Research Paper

\title{
Driver Assistance System to Encourage Appropriate Longitudinal Behavior Considering Pre-preceding Vehicle Information
}

\author{
Yoshitaka Marumo $^{1)}$ Takashi Nakano $^{2)}$ Hironori Suzuki ${ }^{3)}$ \\ 1) Nihon University, College of Industrial Technology \\ 1-2-1 Izumi-cho, Narashino-shi, Chiba 275-8575, Japan (E-mail: marumo.yoshitaka@nihon-u.ac.jp) \\ 2) Graduate School of Nihon University \\ 1-2-1 Izumi-cho, Narashino-shi, Chiba 275-8575, Japan \\ 3) Nippon Institute of Technology, School of Engineering \\ Gakuendai 4-1, Miyashiro Town, Saitama 345-8501 Japan
}

Received on April 28, 2015

Presented at the Second International Symposium on Future Active Safety Technology toward zero traffic accidents (FAST-zero '13), on September 25, 2013

\begin{abstract}
This study proposes a driver assistance system that provides an appropriate pedal operation by considering information on the pre-preceding vehicle. The system calculates the risk of collision by taking into account not only the preceding vehicle, but also the pre-preceding vehicle ahead of the preceding car. The computed numerical risk is then translated into the visual interface that assists the driver to make an appropriate acceleration and deceleration in carfollowing. Several participants who participated in driving simulator experiments were instructed to follow a preceding as well as a visible pre-preceding vehicles with and without the driver assistance system. It was found that the assistance system succeeded in reducing the relative velocity with the pre-preceding vehicle and in eliminating the unnecessary acceleration and deceleration of the following vehicle. Safety and fuel economy were also significantly improved by introducing the proposed system.
\end{abstract}

KEY WORDS: safety, active safety, driving support, pre-preceding vehicle, predicted driving behavior, following characteristics, pedal operation [C1]

\section{Introduction}

Driver assistance systems, which encourage drivers to improve their driving behavior by indicating driving information to drivers, have been developed and commercialized. A fuel economy meter and the indication of collision risk are well known as the fundamental measures for drivers to make a eco-friendly (1),(2) and safe driving ${ }^{(3),(4)}$. In order to improve the fuel economy and to minimize the collision risk to the preceding vehicle, the driver should predict appropriate driving behavior such as the earlier deceleration by using the information on the ahead of the preceding vehicle.

It is obvious that the fuel economy depends on the vehicle states and drivers' characteristics. Earlier release of gas pedal by utilizing the information on the behavior of preceding vehicle is effective to improve the fuel economy ${ }^{(5)}$. In addition, the collision risk to the preceding vehicle depends on the relation between the preceding and following vehicles. However, if the preceding vehicle shows an inappropriate behavior, suppressing the collision risk to the preceding vehicle does not contribute to safety driving $^{(6)}$. Therefore, predicting appropriate driving behavior of the following vehicle is more important by using the information not only on the preceding vehicle but also on the pre-preceding vehicle. Recently, the assistance system, which alerts the occurrence of emergency deceleration of the pre-preceding vehicle, to the driver has been developed ${ }^{(7)}$.
This study proposes the evaluation index to predict appropriate driving behavior by using the information not only on the preceding vehicle but also on the pre-preceding vehicle. The driving simulator experiments were carried out to evaluate the driver assistance system that encourages an appropriate longitudinal driving behavior by indicating the proposed evaluation index to the driver in real-time.

\section{Evaluation Index of Predicted Driving Behavior}

A three-car platoon which consists of pre-preceding $\left(1^{\text {st }}\right)$, preceding $\left(2^{\text {nd }}\right)$ and the following $\left(3^{\text {rd }}\right)$ vehicles is assumed as depicted in Fig.1. Denoting $i$ as the vehicle number, $v_{i}$ and $d_{i+1}$ are defined as the velocity of the $i^{\text {th }}$ vehicle and the headway distance between the $i^{\text {th }}$ and the $i+1^{\text {th }}$ vehicles. For example, $d_{23}$ corresponds to the headway distance between the preceding and the following vehicles.

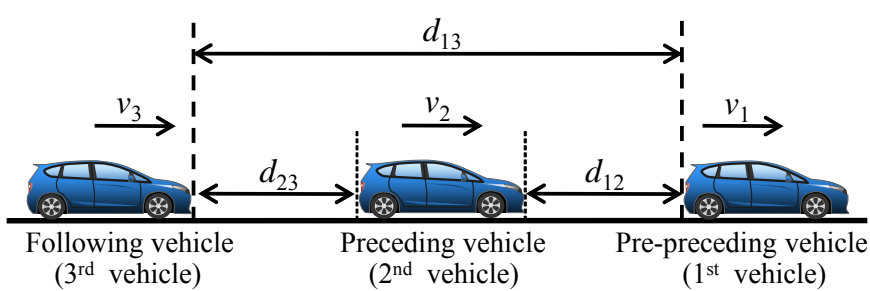

Fig. 1 Three-car platoon. 
One of the evaluation indices for the collision risk to the preceding vehicle ${ }^{(8)}$ is the Risk Perception (RP) ${ }^{(9)}$. The RP is defined as the weighted sum of the inverse of Time To Collision (TTC) and the inverse of the Time HeadWay (THW) as defined in Eq. (1). This index represents the driver's subjective risk to the preceding vehicle.

$\mathrm{RP}=\frac{a}{\mathrm{THW}_{23}}+\frac{b}{\mathrm{TTC}_{23}}=\frac{a v_{3}}{d_{23}}+\frac{b\left(v_{3}-v_{2}\right)}{d_{23}}$

Here, $\mathrm{THW}_{23}$ and $\mathrm{TTC}_{23}$ are the time headway and the time to collision of the following $\left(3^{\text {rd }}\right)$ vehicle to the preceding $\left(2^{\text {nd }}\right)$ vehicle, respectively. Coefficients $a$ and $b$ are equal to 1 and 5 according to the study by Kondoh, et al. ${ }^{(9)}$.

The proposed evaluation index for the predicted driving behavior considering the pre-preceding $\left(1^{\text {st }}\right)$ vehicle is based on the RP. Suppressing the relative velocity to the pre-preceding vehicle reduces the unnecessary acceleration and deceleration. Therefore, TTC, which is effected by the relative velocity, is applied to the pre-preceding vehicle. In addition, in order to prevent the collision to the preceding vehicle, THW is still applied to the preceding vehicle. The coefficient $b$ in Eq. (1) is twice because the TTC, which is proportional to the headway distance, is applied to the pre-preceding vehicle. The novel index, which is referred to as the PRE3 (PREdiction by PRE-PREceding vehicle), is defined as follows;

PRE3 $=\frac{a}{\mathrm{THW}_{23}}+\frac{2 b}{\mathrm{TTC}_{13}}=\frac{a v_{3}}{d_{23}}+\frac{2 b\left(v_{3}-v_{1}\right)}{d_{13}}$

where $\mathrm{TTC}_{13}$ is the time to collision of the following $\left(3^{\text {rd }}\right)$ vehicle to the pre-preceding $\left(1^{\text {st }}\right)$ vehicle.

\section{Driving Simulator Experiments}

\subsection{Experimental Method}

In order to examine the effects of the driver assistance system by indicating the PRE3 to the driver in real time, the driving simulator experiments are conducted. Experimental participants are required to follow the two vehicles, i.e. a prepreceding and a preceding vehicles, with and without the assistance system. This study uses the CarSim DS 8.1.1 (Mechanical Simulation Corporation) ${ }^{(10)}$ as shown in Fig. 2 for the driving simulator experiments.

The velocity pattern of the pre-preceding and preceding vehicles are assumed as shown in Fig.3. The pre-preceding vehicle repeats the acceleration and deceleration with $1 \mathrm{~m} / \mathrm{s}^{2}$ and travels between $10 \mathrm{~m} / \mathrm{s}(36 \mathrm{~km} / \mathrm{h})$ and $15 \mathrm{~m} / \mathrm{s}(54 \mathrm{~km} / \mathrm{h})$. The following algorithm of the preceding vehicle to follow the prepreceding vehicle is based on the PID control. The PID controller compensates the heaway distance error between the actual distance and the desired heaway distance, which is calculated by multiplying the vehicle velocity and the desired time headway (1.5s). The feedback gains for the PID control are adjusted to simulate the general driver's following characteristics.

The driving course is a straight corridor. Oncoming vehicles appear at random during the experiments. The pre-preceding vehicle is a tall minivan and the preceding vehicle is placed at the

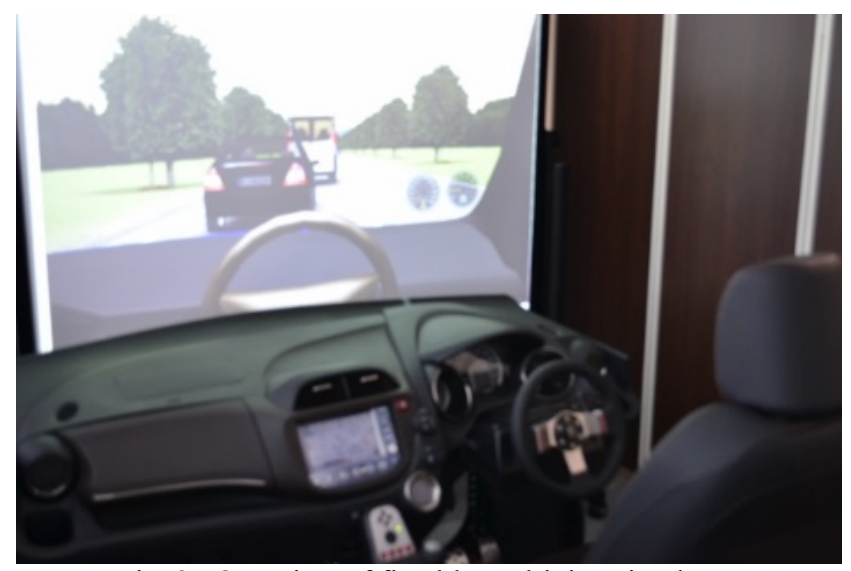

Fig. 2 Overview of fixed-base driving simulator.

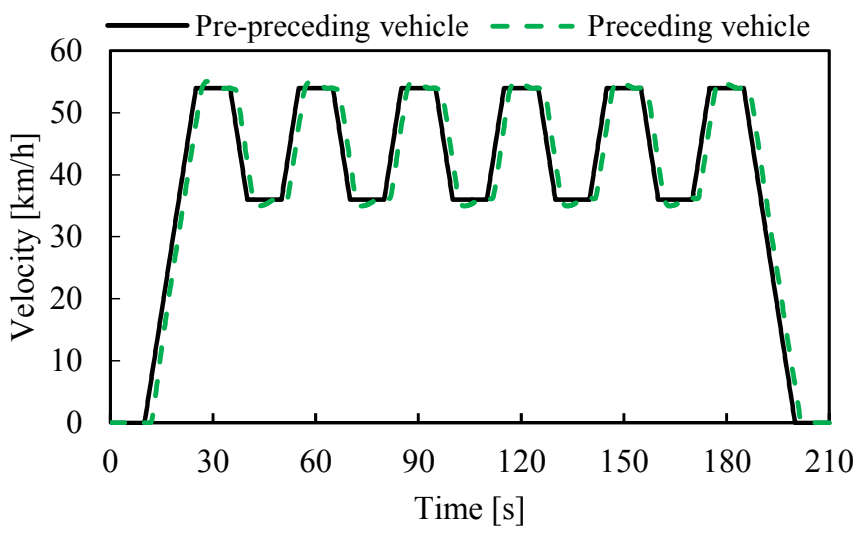

Fig. 3 Velocity profile of pre-preceding and preceding vehicles.

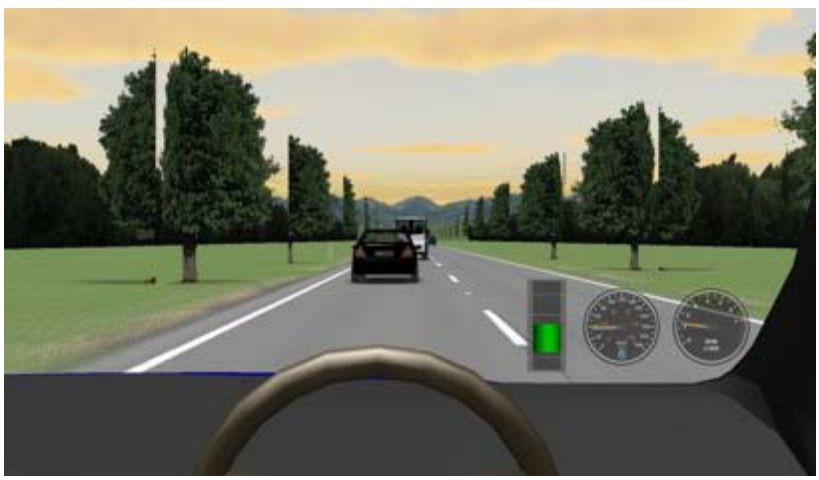

(a) Indicator in driver's view

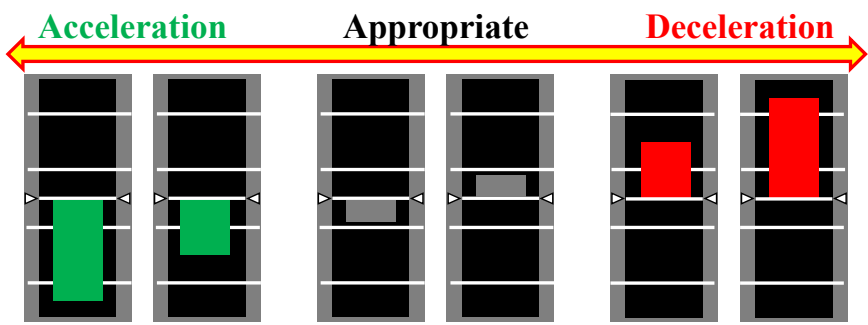

(b) Indicator bar and required operation

Fig. 4 Graphical image of PRE3 indicator. 
left side on the road to make the driver recognize the prepreceding vehicle easily even without the assistance system.

Figure 4 illustrates the image of the PRE3 indicator. The bar length and colors of the indicator correspond to the PRE3 value. The bar colors consist of green, red and gray as shown in Fig. 4(b). The neutral value of the PRE3 is set to $0.5 \mathrm{~s}^{-1}$, and this value means that the time headway to the preceding vehicle $\mathrm{THW}_{23}$ is equal to $2 \mathrm{~s}$ if the relative velocity to the pre-preceding vehicle is $0 \mathrm{~m} / \mathrm{s}$ If the following vehicle velocity $v_{3}$ is slower than the prepreceding vehicle velocity $v_{1}$ or the following vehicle is away from the preceding vehicle (longer $\mathrm{THW}_{23}$ ), the indicator bar is extended downward and is colored in green. This is supposed to encourage the driver to accelerate to increase the PRE3 value. On the other hand, if the velocity of the following vehicle $v_{3}$ is faster than that of the pre-preceding vehicle $v_{1}$ or the following vehicle is close to the preceding vehicle (shorter $\mathrm{THW}_{23}$ ), the indicator bar is extended upward and is colored in red. The driver should decelerate to reduce the PRE3 value. If the driver performs the appropriate driving behavior, the indicator maintains the gray bar.

Nine males, whose ages ranged from 20 to 23 years old, participated in the simulator experiments. Their driving experiences are between nine and 34 months, with an average experimence of 22 months. All the participants gave their written informed consent before the experiments. The test drive was conducted to familiarize themselves with the simulator driving and the behavior of the PRE3 indicator. The participants are to experience the trials with the assistance system before the experiments without the assistance. These two driving conditions, i.e. with and without the assistance system, were repeated twice, to make all four trials for each participant.

The participants were instructed to follow the rules and regulations below; "Look at the pre-preceding vehicle behavior carefully and predict appropriate driving behavior by the earlier acceleration and deceleration." "Drive the car so as to maintain the indicator bar stay in the gray color." "Even without the assistance system, follow the two vehicles ahead just like your car was equipped with the assistance system." The last instruction is given in order to evaluate the effect of the proposed system as precise as possible.

\subsection{Experimental Results}

\subsubsection{Driving behavior of following vehicle}

Figure 5 illustrates an example of the speed variation of the following vehicle (2nd trial by participant A). The solid line indicates the following vehicle velocity with the assistance system and the dashed line is without the assistance system. The driver with the assistance system reacts the velocity change of the prepreceding vehicle, while the driver without the assistance delays his acceleration to the pre-preceding vehicle. The assistance system prevents the delay of velocity change of the pre-preceding vehicle and reduces the relative velocity to the pre-preceding vehicle. This effect contributes the suppression of unnecessary acceleration and deceleration.

The RMS of the relative velocity to the pre-preceding vehicle is depicted in Fig. 6. This figure shows the average of 18 trials (total of the two conditions for the nine participants). The assistance system reduces the RMS of the relative velocity to the

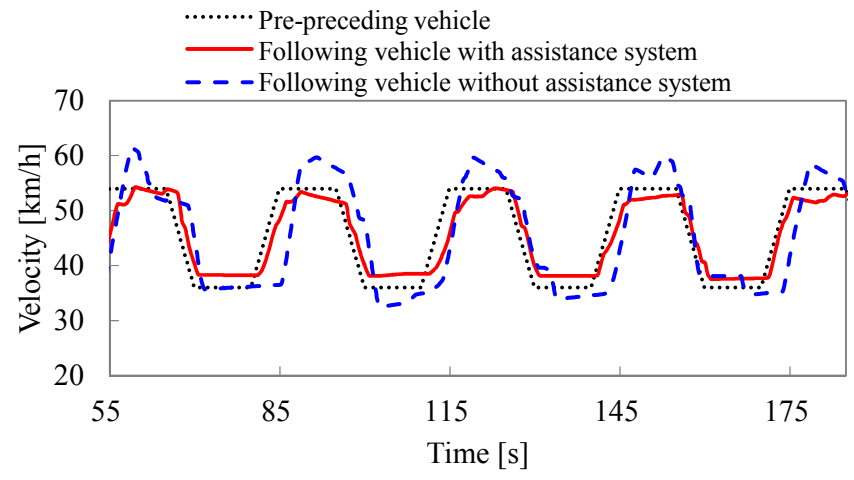

Fig. 5 Velocity of following vehicle (2nd trial by participant A).

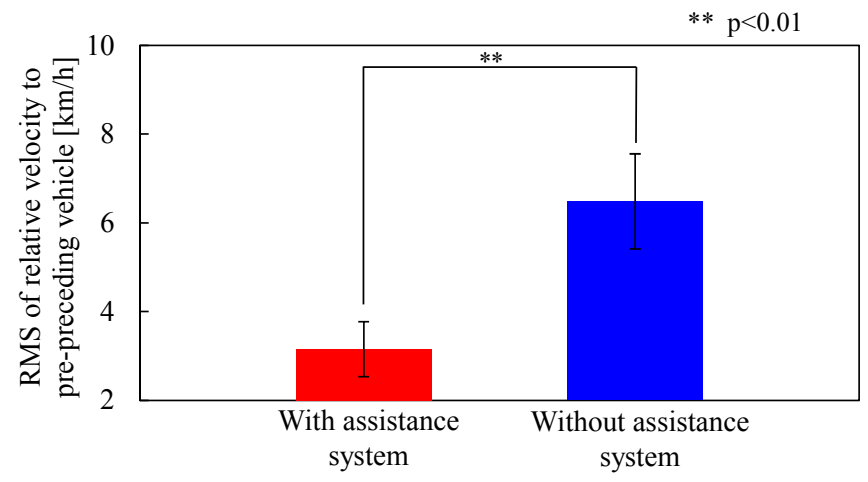

Fig. 6 RMS of relative velocity to pre-preceding vehicle.

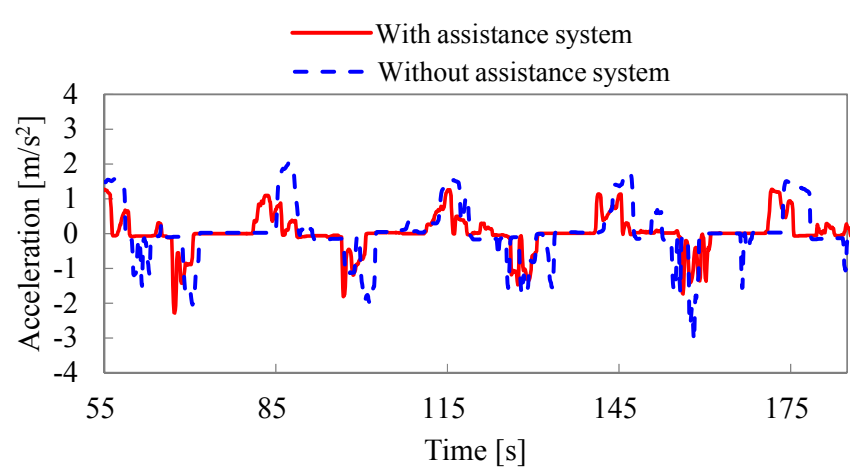

Fig. 7 Acceleration of following vehicle (2nd trial by participant A).

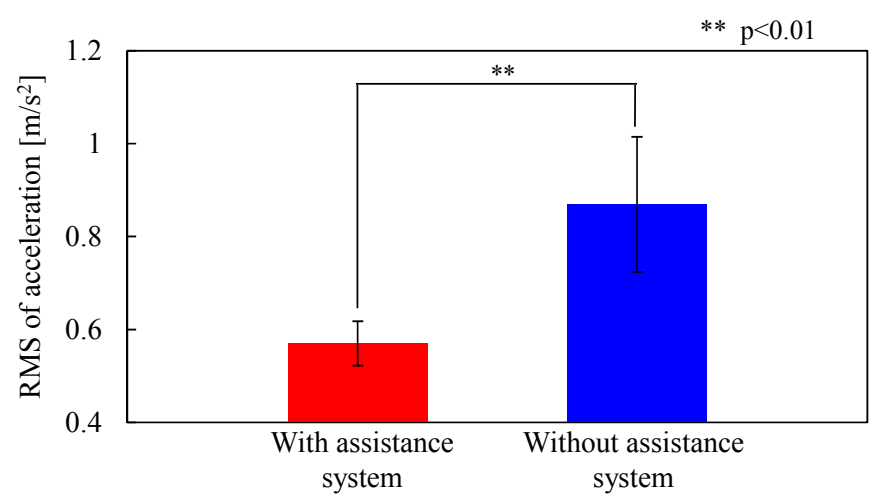

Fig. 8 RMS of acceleration. 


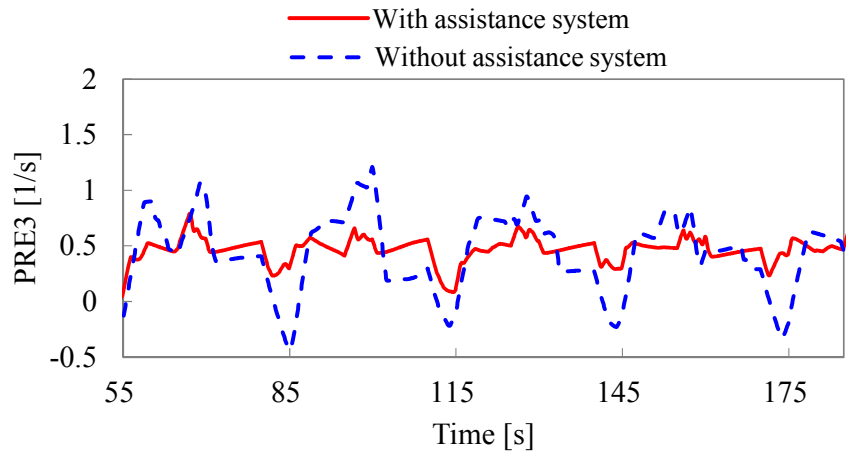

Fig. 9 PRE3 of following vehicle (2nd trial by participant A).

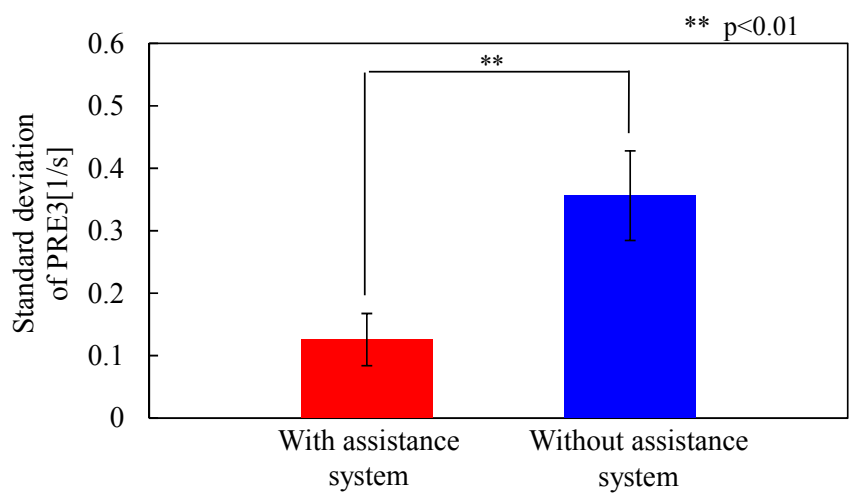

Fig. 10 Standard deviation of PRE3.

pre-preceding vehicle with a significant difference under the $1 \%$ confidential level. This means that the assistance system makes the driver recognize the velocity change of the pre-preceding vehicle and prevents the unnecessary acceleration and deceleration.

The acceleration variation of the following vehicle (2nd trial by participant A) is shown in Fig. 7. The line types are same as the Fig. 5. The acceleration response with the assistance system is earlier than that without the assistance system. In addition, the maximum and minimum acceleration are suppressed by the assistance system.

Figure 8 shows the RMS of the following vehicle acceleration, averaged by 18 trials. The assistance system reduces the RMS of acceleration with a significant difference under the $1 \%$ confidential level.

\subsubsection{Evaluation indices}

Figure 9 illustrates the PRE3 variation of the following vehicle ( 2 nd trial by participant $A$ ). The driver with the assistance system maintains the PRE3 around $0.5 \mathrm{~s}^{-1}$, while the driver without the assistance system makes the PRE3 fluctuate around $0.5 \mathrm{~s}^{-1}$. The assistance system reduces the fluctuation of the PRE3.

Thought the mean of the PRE3 with and without the assistance system has no significant difference, its standard deviation shows the large variation as depicted in Fig. 10. The assistance system suppresses the standard deviation of the PRE3 with a significant difference under the $1 \%$ confidential level.

Figure 11 illustrates the Risk Perception variation of the following vehicle ( 2 nd trial by participant A). The RP with the

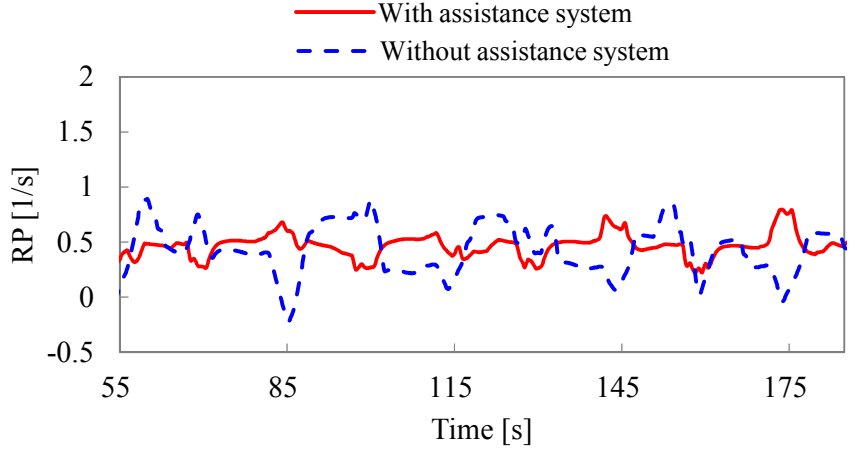

Fig. 11 Risk Perception of following vehicle (2nd trial by participant A).

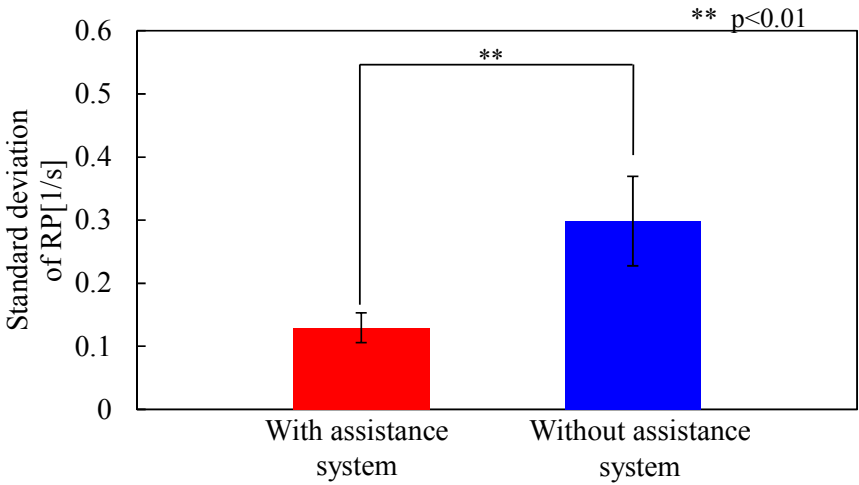

Fig. 12 Standard deviation of RP.

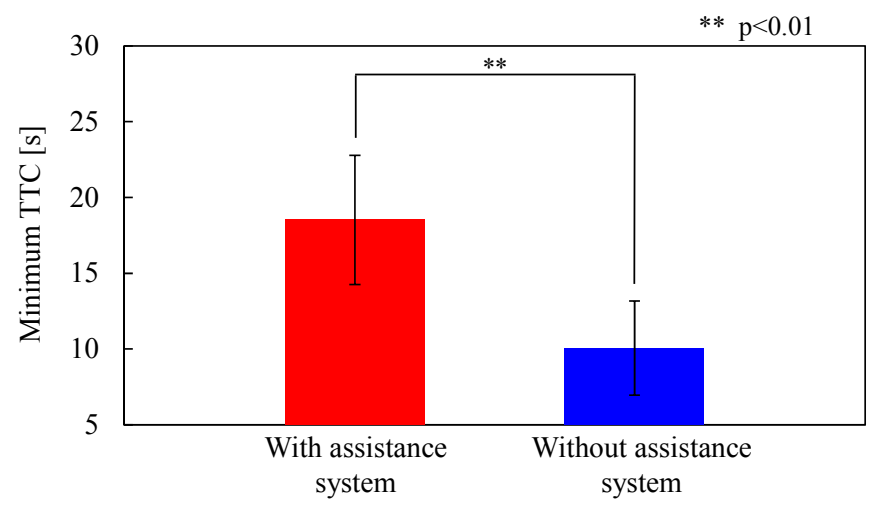

Fig. 13 Minimum value of TTC.

assistance system is maintained around $0.5 \mathrm{~s}^{-1}$, while the RP without the assistance system is fluctuated around $0.5 \mathrm{~s}^{-1}$. The RP fluctuation is also reduced by the assistance system.

The standard deviation of the RP, averaged by 18 trials, is shown in Fig. 12. The assistance system also suppresses the standard deviation of the RP with a significant difference under the $1 \%$ confidential level.

These effects can be seen in the minimum value of the TTC to the preceding vehicle as shown in Fig. 13. The assistance system increases the minimum value with a significant difference under the $1 \%$ confidential level and realizes the safety driving to the preceding vehicle. 


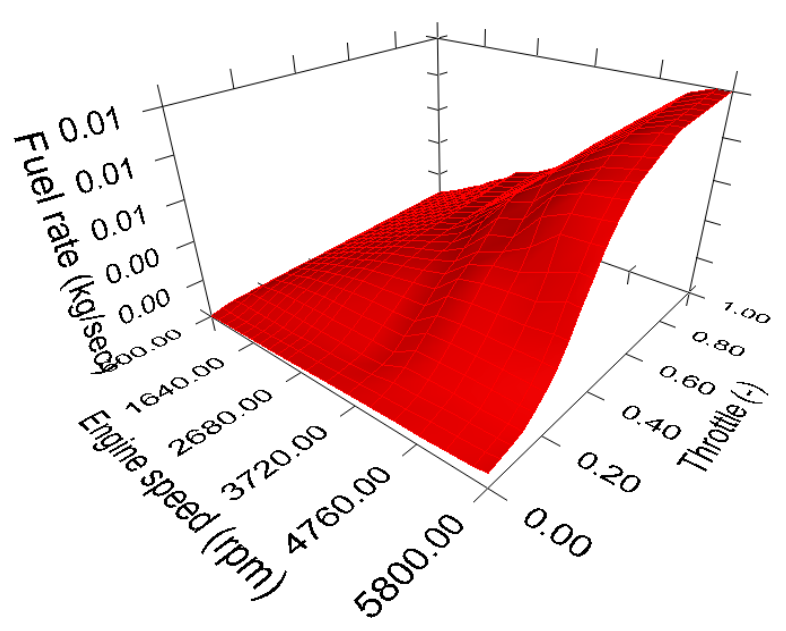

Fig. 14 Fuel consumption rate map ${ }^{(10)}$.

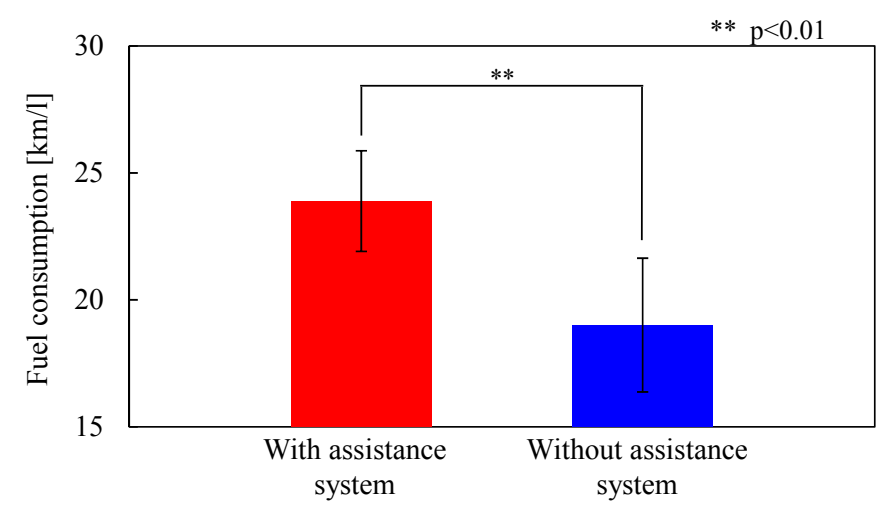

Fig. 15 Fuel consumption.

\subsubsection{Fuel economy}

The fuel consumption is also calculated based on the fuel consumption rate map in the software ${ }^{(10)}$ as shown in Fig. 14. The output of the engine this study uses is $150 \mathrm{~kW}$, and the density of the gasoline is $0.77 \mathrm{~kg} / \mathrm{l}$.

Figure 15 illustrates the fuel economy of the following vehicle. The assistance system suppresses the fuel economy with a significant difference under the $1 \%$ confidential level.

The proposed assistance system improves not only safety to the preceding vehicle, but also fuel economy of the following vehicle.

\section{Conclusion}

This study proposes a system to assist an appropriate longitudinal driving by considering information not only on the preceding vehicle but also on the pre-preceding vehicle. The driving simulator experiments are carried out to investigate the effects of the assistance system, which indicates the proposed evaluation index, on the driving behavior.

The driving simulator experiments revealed that indicating the PRE3 reduces the unnecessary acceleration and deceleration to minimize the unexpected deterioration of traveling speed. The change of the RP is also suppressed, and the assistance system makes it possible to suppress the collision risk to the preceding vehicle. In addition, indicating the PRE3 also reduces the fuel consumption of the following vehicle.

This study assumes that a vehicle is equipped with some special state-of-the-art technologies that directly measures the quantity of state of the pre-preceding vehicle ${ }^{(7)}$. The inter-vehicle communication can be a solution to realize the assistance system considering the pre-preceding vehicle ${ }^{(11)}$. In addition, if the intermediate (the preceding) vehicle among three cars directly measures the relative states with its forward (the pre-preceding) and backward (the following) vehicles, the sensing problem can be solved by indicating the evaluation index at the rear-end of the intermediate vehicle ${ }^{(12)}$

\section{References}

(1) T. Hiraoka, S. Nishikawa and H. Kawakami: Driverassistance system to encourage spontaneous eco-driving behavior, Proceedings of 18th World Congress on Intelligent Transport Systems, CD-ROM (2011).

(2) T. Wada, K. Yoshimura, S. Doi, H. Youhata and K. Tomiyama: Proposal of An Eco-Driving Assist System Adaptive to Driver's Skill, Proceedings of the IEEE International Conference on Intelligent Transportation Systems, p. 1880-1885, (2011).

(3) K. Shidoji, T. Kumagai, S. Watanabe, Y. Matsuki and T. Nakamura: Development of safe driving promotion system by displaying driving scores and rankings, International Journal of ITS Research, Vol. 5, No. 1, p. 55-62, (2007).

(4) S. Takada, T. Hiraoka and H. Kawakami: Effect of forward obstacles collision warning system based on deceleration for collision avoidance on driving behavior, Proceedings of 19th World Congress on Intelligent Transport Systems, CD-ROM (2012).

(5) M.A.S Kamal, M. Mukai, J. Murata and T. Kawabe: Ecological driver assistance system using model-based anticipation of vehicle-road-traffic information, IET Intelligent Transportation systems, Vol. 4, No. 4, p. 244-251, (2010).

(6) H. Suzuki and T. Katayama: Controlling Vehicle Platoon to Alleviate Shockwave Propagation, Proceedings of the AsiaPacific Automotive Engineering Conference 2013 (APAC17), SAE Technical Paper 2013-01-0022 (2013).

(7) Nissan Motor Company: Predictive Forward Collision Warning, http://www.nissanglobal.com/EN/TECHNOLOGY/OVERVIEW/predictive.ht $\mathrm{ml}$ (accessed on September 18, 2015)

(8) S. Kitajima, Y. Marumo, T. Hiraoka and M. Itoh: Comparison of Evaluation Indices concerning Estimation of Driver's Risk Perception -Risk perception of rear-end collision to a preceding vehicle-, Review of Automotive Engineering, Vol. 30, No. 2, p. 191-198 (2009).

(9) T. Kondoh, T. Yamamura, S. Kitazaki, N. Kuge and E.R Boer: Identification of Visual Cues and Quantification of Drivers' Perception of Proximity Risk to the Lead Vehicle in Car-Following Situations; Journal of Mechanical Systems for Transportation and Logistics, Vol. 1, No. 2, p. 170-180 (2008).

(10) Mechanical Simulation Corporation: Driving Simulator reference manual, (2012).

(11) T. Nakano, Y. Marumo, H. Suzuki and T. Kawai: Experimental evaluation of assistance system to predict driving behavior considering pre-preceding vehicle 
information (in Japanese with English summary), Transactions of the Japan Society of Mechanical Engineers, Vol. 81, No. 828, p. 15-00124 (2015).

(12) T. Nakano, Y. Marumo and H. Suzuki: Driver assistance system indicating information on predicted longitudinal maneuver at rear-end of preceding vehicle (in Japanese with English summary), Transactions of the Japan Society of Mechanical Engineers, Vol. 81, (2015) (accepted). 